\title{
Thermodynamic interaction between two discrete systems in non-equilibrium
}

\author{
Wolfgang Muschik ${ }^{1, *}$ and Arkadi Berezovski ${ }^{2}$ \\ ${ }^{1}$ Institut für Theoretische Physik, Technische Universität Berlin, Germany \\ ${ }^{2}$ Centre for Nonlinear Studies, Institute of Cybernetics at Tallinn Technical University, \\ Estonia \\ ${ }^{*}$ Corresponding author \\ Institut für Theoretische Physik, Technische Universität Berlin, Hardenbergstr. 36, \\ D-10623 Berlin, Germany \\ E-mail: muschik@physik.tu-berlin.de
}

Communicated by B. Andresen, Copenhagen, Denmark and K.-H. Hoffmann, Chemnitz, Germany

\begin{abstract}
A systematic description of the interaction between two systems is presented within the framework of thermodynamics of discrete systems. We distinguish between two situations: a contact between an equilibrium system and a non-equilibrium one, and a contact between two non-equilibrium systems. In the second case the state space includes replacement quantities, which reduce the situation to a contact between an equilibrium system and a non-equilibrium one. Interacting discrete systems form a composite (compound) system which is also a discrete one. Thus a compound system can be described as if it were composed of two subsystems, or by ignoring this fact, as a plain single discrete system, whose composition of sub-systems is not taken into consideration. These descriptions cause different degrees of accuracy which are expressed by excess quantities. Such an excess quantity vanishes if the discrete system is really not composed of subsystems. Aim of this paper is to calculate the excess quantities of power and energy exchange and to derive the excess entropy in the special case of an endoreversible compound system.
\end{abstract}

\section{Introduction}

There are two different descriptions of thermodynamic systems: the field formulation and the description as a discrete (or lumped) system. The field formulation or continuum thermodynamics deals with balance equations [1-4], which model together with the constitutive equations (equations of state) and the initial and boundary conditions the process going on in the system. 
After having inserted the constitutive equations into the balance equations, we obtain a system of partial differential equations whose analytical solutions can be calculated only in sufficiently simple cases. In numerous practical applications, these continuous models are replaced by approximations usually obtained by means of finite differences or finite elements. Calculations are carried out after having chosen an appropriate algorithm with respect to the problems of stability and convergence. Consequently, for practical reasons it seems to be more convenient to have a direct description of the coupled thermodynamic behaviour of a finite number of interacting elements or cells. The introduction of the concept of discrete systems [5] gives a simple and effective method to describe thermodynamics and interactions of elements in non-equilibrium. But here we encounter the analogous difficulties which appear in classical thermodynamics, as noted by Truesdell and Bharatha [6], "the formal structure of classical thermodynamics describes the effects of changes undergone by some single body. While it allows these effects for one body to be compared with corresponding effects for another body, it does not represent the effects associated with two bodies simultaneously or in any way conjointly." The interactions between a discrete system and its equilibrium environment is well known [7], but the general interaction between two discrete non-equilibrium systems has not been investigated up to now and is shortly discussed here.

To describe the interaction between two discrete systems, we note that interacting systems always form a composite system. It is clear that the thermodynamic description of a composite system should be consistent with that of its interacting subsystems. Also clear is that the accuracy of description depends on the information one has of the discrete system: if a compound system is described as being non-composite, the accuracy of description is lower than taking its composition into account. The thermodynamic consistency of these two different descriptions is achieved by introducing excess quantities which vanish if the discrete system is non-composite.

The paper is organized in the following way: In Section 2 the main features of thermodynamics of discrete systems are remembered. Replacement quantities, introduced in Section 3, are necessary for the description of a non-equilibrium system in a non-equilibrium environment. Further, we have to consider the interaction between two systems which are coupled to each other and therefore are forming a composite system. The different descriptions of discrete systems with respect to their composition are investigated in Section 4. The thermodynamic consistency between these descriptions is achieved by introducing excess quantities of energy, entropy, and power and energy exchanges. Main conclusions are given in the summary.

\section{Equilibrium/non-equilibrium contacts}

\subsection{Contact quantities}

By definition, a discrete (or lumped) system is a region $G \in \mathbf{R}^{3}$ in space separated by a contact surface $\mathscr{F}$ from its environment $G^{*}$ [7]. The interaction between $G$ and $G^{*}$ can be described by exchange quantities. We call $G$ a Schottky system [8], if the inter- 


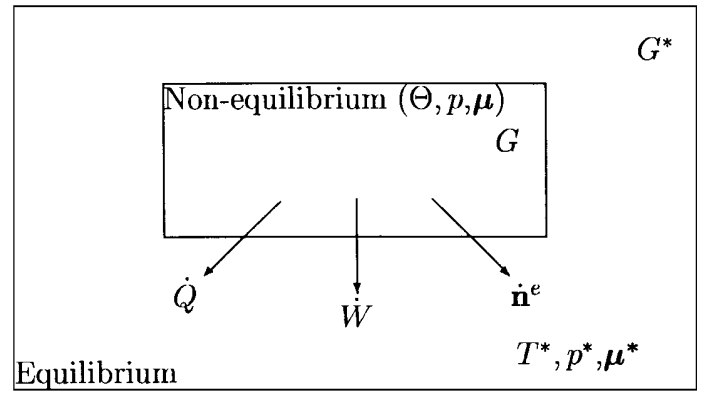

Figure 1 General structure of Schottky systems.

action with its environment consists of heat exchange $\dot{Q}$, of power exchange $\dot{W}$, and of mass exchange described by time rates of numbers of moles $\dot{\mathbf{n}}^{e}$ of the different species (Fig. 1).

The exchange quantities $\dot{Q}, \dot{W}$, and $\dot{\mathbf{n}}^{e}$ determine intensive non-equilibrium contact quantities, namely the contact temperature $\Theta$, the dynamic pressure $p^{1}$, and the $d y$ namic chemical potentials $\boldsymbol{\mu}$ by different defining inequalities [7]. These inequalities and their corresponding constraints are:

$$
\begin{aligned}
& \left(\frac{1}{\Theta}-\frac{1}{T^{*}}\right) \dot{Q}\left(\Theta, T^{*}\right) \equiv\left(\frac{1}{\Theta}-\frac{1}{T^{*}}\right) \dot{Q} \geq 0, \quad\left(\dot{V}=0, \dot{\mathbf{n}}^{e}=\mathbf{0}\right), \\
& \left(p-p^{*}\right) \dot{V}\left(p, p^{*}\right) \equiv\left(p-p^{*}\right) \dot{V} \geq 0, \quad\left(\dot{Q}=0, \dot{\mathbf{n}}^{e}=\mathbf{0}\right), \\
& \left(\boldsymbol{\mu}^{*}-\boldsymbol{\mu}\right) \cdot \dot{\mathbf{n}}^{e}\left(\boldsymbol{\mu}, \boldsymbol{\mu}^{*}\right) \equiv\left(\boldsymbol{\mu}^{*}-\boldsymbol{\mu}\right) \cdot \dot{\mathbf{n}}^{e} \geq 0, \quad(\dot{Q}=0, \dot{V}=0) .
\end{aligned}
$$

Here $V$ is the volume of the discrete system $G, T^{*}$ is the thermostatic temperature of the equilibrium environment $G^{*}, p^{*}$ its equilibrium pressure, and $\boldsymbol{\mu}^{*}$ its equilibrium chemical potentials.

The contact temperature is the dynamical analogue to the thermostatic temperature $[9,10]$. It is a quantity belonging to the whole contact surface: hence its name is contact temperature. When changing the contact surface, the contact temperature changes, too, in general. The interpretation of the contact temperature is as follows: From Eq. (1) it is evident, that the heat exchange $\dot{Q}$ and the bracket always have the same sign [11]. We now presuppose that there exists exactly one equilibrium environment for each arbitrary state of a discrete system for which the net heat exchange between them vanishes. Consequently, the defining inequality (1) determines the contact temperature $\Theta$ of the system as that thermostatic temperature $T^{*}$ of the system's environment for which this net heat exchange vanishes and no power and material exchanges occur. The same interpretation holds true for the dynamical pressure $p$ and the dynamical chemical potentials $\boldsymbol{\mu}$ with respect to the net rate of the volume

\footnotetext{
${ }^{1}$ As a special simple example for demonstration.
} 
and to the net rate of each mole number due to external material exchange. According to the defining inequalities (1) to (3) there exists an equilibrium environment for which the net heat exchange, the net power exchange, and the net external material exchange for each component between $G$ and $G^{*}$ vanish. In this case, the intensive variables $T^{*}, p^{*}, \boldsymbol{\mu}^{*}$ of this environment define the values of the non-equilibrium contact quantities $\Theta, p, \boldsymbol{\mu}$ of the system in consideration.

In all cases the constraints mentioned in the brackets in Eqs. (1) to (3) have to be taken into consideration for each defining inequality. These defining inequalities are operational definitions of the contact quantities based on measuring rules which are expressed by the constraints mentioned in Eqs. (1-3). The contact quantities $\Theta, p$, and $\boldsymbol{\mu}$ themselves are of course independent of these constraints and of the special values of $T^{*}, p^{*}$, and $\boldsymbol{\mu}^{*}$. Only for measuring them do the constraints have to be taken into consideration. Consequently, $\Theta, p$, and $\boldsymbol{\mu}$ are generally defined for Schottky systems.

Contact quantities satisfy constitutive equations

$$
\begin{aligned}
& \dot{Q}=F\left(\frac{1}{\Theta}-\frac{1}{T^{*}}\right), \quad\left(\dot{V}=0, \dot{\mathbf{n}}^{e}=\mathbf{0}\right), \\
& \dot{V}=G\left(p-p^{*}\right), \quad\left(\dot{Q}=0, \dot{\mathbf{n}}^{e}=\mathbf{0}\right), \\
& \dot{\mathbf{n}}^{e}=\mathbf{H}\left(\boldsymbol{\mu}^{*}-\boldsymbol{\mu}\right), \quad(\dot{Q}=0, \dot{V}=0) .
\end{aligned}
$$

According to Eqs. (1-3) and their presupposed continuity the constitutive functions $F, G$, and $\mathbf{H}$ have the property

$$
\begin{array}{cc}
y F(y) \geq 0, & F(0)=0, \\
y G(y) \geq 0, & G(0)=0, \\
\mathbf{y} \cdot \mathbf{H}(\mathbf{y}) \geq 0, & \mathbf{H}(\mathbf{0})=\mathbf{0} .
\end{array}
$$

Beyond these properties we additionally demand the strict monotony of $F, G$, and $\mathbf{H}$

$$
\begin{aligned}
& y>y^{+} \Rightarrow F(y)>F\left(y^{+}\right) \text {and } G(y)>G\left(y^{+}\right), \\
& \mathbf{y}>\mathbf{y}^{+} \Rightarrow \mathbf{H}(\mathbf{y})>\mathbf{H}\left(\mathbf{y}^{+}\right) .
\end{aligned}
$$

The non-equilibrium state space of a discrete system in a rest frame can be chosen as follows [3]:

$$
Z=\left\{V, \mathbf{n}, U, \Theta, \xi ; T^{*}, p^{*}, \boldsymbol{\mu}^{*}\right\}
$$

Here $U$ is the internal energy of the system, and $\boldsymbol{\xi}$ are other variables in connection to irreversible processes going on in the system. Up to now it has not been necessary 


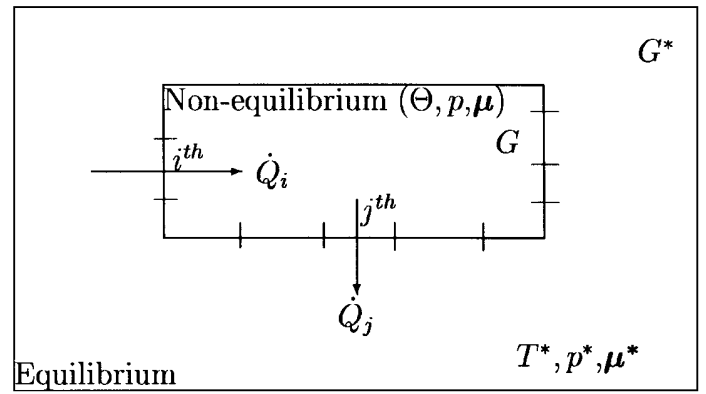

Figure 2 Division of the contact surface.

to specify these variables. The dynamic pressure and the dynamical chemical potentials, and according to Eqs. (4) and (5) $G$ and $\mathbf{H}$, are constitutive equations on $Z$ (see section 4.2, Eq. (65)). Thus, the complete description of the non-equilibrium state of a discrete system includes its contact quantities and the intensive variables of the environment as parameters which satisfy a Gibbs-Duhem equation; that means, one of the $\boldsymbol{\mu}^{*}$ can be expressed by the other ones and by $T^{*}$ and $p^{*}$.

\subsection{Partial contact quantities}

The contact surface $\mathscr{F}$ between the system and its environment can be arbitrarily divided into parts $\mathscr{F}_{i}^{+}$and $\mathscr{F}_{j}^{-}$(Fig. 2) defined by the following properties $[12,13]$ :

$$
\begin{aligned}
& \mathscr{F}^{+}=\bigcup_{i} \mathscr{\mathscr { F }}_{i}^{+}, \quad \mathscr{F}_{i}^{+} \cap \mathscr{F}_{k}^{+}=\varnothing, \quad i \neq k, \\
& \mathscr{F}^{-}=\bigcup_{j} \mathscr{F}_{j}^{-}, \quad \mathscr{F}_{j}^{-} \cap \mathscr{F}_{k}^{-}=\varnothing, \quad j \neq k, \\
& \mathscr{F}=\mathscr{F}^{+} \cup \mathscr{F}^{-}, \quad \mathscr{F}^{+} \cap \mathscr{F}^{-}=\varnothing .
\end{aligned}
$$

The denotion of the partial surfaces is determined by the signs of the heat exchanges: $\dot{Q}_{i}^{ \pm}$are the heat exchanges through $\mathscr{F}_{i}{ }^{ \pm}$.

$$
\begin{array}{ll}
\mathscr{F}_{i}^{+}: \quad \dot{Q}_{i}^{+} \geq 0, \quad \dot{Q}^{+}:=\sum_{i} \dot{Q}_{i}^{+} \geq 0, \\
\mathscr{F}_{j}^{-}: \quad \dot{Q}_{j}^{-}<0, \quad \dot{Q}^{-}:=\sum_{j} \dot{Q}_{j}^{-}<0, \quad \dot{Q}^{+}+\dot{Q}^{-}=\dot{Q} .
\end{array}
$$

We now consider a special state of $G$ which we call partial equilibrium with respect to $p$ and $\boldsymbol{\mu}$. This partial equilibrium of $G$ is defined by

$$
\mathscr{F}_{k}^{ \pm}: \quad p_{k}=p^{*}, \quad \boldsymbol{\mu}=\boldsymbol{\mu}^{*}, \quad \text { for all } k .
$$


The partial equilibrium with respect to $p$ and $\boldsymbol{\mu}$ means that there are no net power and net material exchanges through each of the partial surfaces $\mathscr{F}_{k}^{ \pm}$according to Eqs. (2) and (3). Only heat exchanges occur. Consequently, the heat exchanges satisfy the inequalities which belong to the partial contact surfaces according to Eq. (1):

$$
\begin{array}{ll}
\mathscr{F}_{i}^{+}: \quad \dot{Q}_{i}^{+}\left(\frac{1}{\Theta_{i}^{+}}-\frac{1}{T^{*}}\right) \geq 0, \quad\left(p_{i}=p^{*}, \boldsymbol{\mu}_{i}=\boldsymbol{\mu}^{*}\right), \\
\mathscr{F}_{j}^{-}: \quad \dot{Q}_{j}^{-}\left(\frac{1}{\Theta_{j}^{-}}-\frac{1}{T^{*}}\right) \geq 0, \quad\left(p_{j}=p^{*}, \boldsymbol{\mu}_{j}=\boldsymbol{\mu}^{*}\right) .
\end{array}
$$

By summing up these inequalities and taking into consideration Eqs. (16) and (17) we obtain by applying the mean value theorem to the sums

$$
\begin{aligned}
& \mathscr{F}^{+}: \quad \sum_{i} \frac{\dot{Q}_{i}^{+}}{\Theta_{i}^{+}}-\frac{\dot{Q}^{+}}{T^{*}} \geq 0 \rightarrow \dot{Q}^{+}\left(\frac{1}{\Theta^{+}}-\frac{1}{T^{*}}\right) \geq 0, \\
& \mathscr{F}^{-}: \quad \sum_{j} \frac{\dot{Q}_{j}^{-}}{\Theta_{j}^{-}}-\frac{\dot{Q}^{-}}{T^{*}} \geq 0 \rightarrow \dot{Q}^{-}\left(\frac{1}{\Theta^{-}}-\frac{1}{T^{*}}\right) \geq 0 .
\end{aligned}
$$

These inequalities are valid for arbitrary $T^{*}$, especially also for

$$
T^{*}=\Theta, \rightarrow \dot{Q}^{+}+\dot{Q}^{-}=0,
$$

which follows from Eq. (1). Hence Eqs. (21) and (22) result in

$$
\begin{aligned}
& \mathscr{F}^{+}: \quad \dot{Q}^{+}\left(\frac{1}{\Theta^{+}}-\frac{1}{\Theta}\right) \geq 0 \rightarrow \Theta^{+} \leq \Theta, \\
& \mathscr{F}^{-}: \quad \dot{Q}^{+}\left(\frac{1}{\Theta}-\frac{1}{\Theta^{-}}\right) \geq 0 \rightarrow \Theta \leq \Theta^{-} .
\end{aligned}
$$

Therefore, we obtain for the partial equilibrium with respect to $p$ and $\mu$

$$
\Theta^{+} \leq \Theta \leq \Theta^{-}, \quad\left(p_{i}=p^{*}, \boldsymbol{\mu}_{i}=\boldsymbol{\mu}^{*}, \text { for all } i\right) .
$$

Because $\Theta, \Theta^{+}$and $\Theta^{-}$are independent of the intensive variables of $G^{*}$ and of $p_{i}$ and $\boldsymbol{\mu}^{*}$, the inequalities (26) are valid in general. This means, that the contact temperature $\Theta$ of $\mathscr{F}$ is always in between the contact temperatures $\Theta^{+}$and $\Theta^{-}$of the partial contact surfaces $\mathscr{F}^{+}$and $\mathscr{F}^{-}$.

From Eqs. (24) and (25) we obtain by Eqs. (16) and (17)

$$
\frac{\dot{Q}^{+}}{\Theta^{+}} \geq \frac{\dot{Q}^{+}}{\Theta}, \quad \frac{\dot{Q}^{-}}{\Theta^{-}} \geq \frac{\dot{Q}^{-}}{\Theta} .
$$


Summing up both the inequalities we obtain by use of Eqs. (21), (22), and (1)

$$
\sum_{k} \frac{\dot{Q}_{k}}{\Theta_{k}} \geq \frac{\dot{Q}}{\Theta} \geq \frac{\dot{Q}}{T^{*}}
$$

Starting out with a partial equilibrium with respect to $\Theta$ and $\boldsymbol{\mu}$, we obtain from Eq. (2) by the same reasoning as before the generally valid inequalities analogously to Eq. (28):

$$
\sum_{k} p_{k} \dot{V}_{k} \geq p \dot{V} \geq p^{*} \dot{V}
$$

Finally, starting out with a partial equilibrium with respect to $\Theta$ and $p$ we obtain from Eq. (3)

$$
\sum_{k} \boldsymbol{\mu}_{k} \cdot \dot{\boldsymbol{n}}_{k}^{e} \leq \boldsymbol{\mu} \cdot \dot{\boldsymbol{n}}^{e} \leq \boldsymbol{\mu}^{*} \cdot \dot{\boldsymbol{n}}^{e}
$$

As to Eq. (4), the partial heat exchanges $\dot{Q}^{+}$and $\dot{Q}^{-}$satisfy constitutive equations which depend on the special partial contact surfaces $\mathscr{F} \pm$ and on $T^{*}$

$$
\begin{aligned}
& \dot{Q}^{ \pm}=F_{ \pm}\left(\frac{1}{\Theta^{ \pm}}-\frac{1}{T^{*}}\right) \\
& F_{+}(x)=0, \quad \text { if } x \leq 0, \quad F_{-}(x)=0, \quad \text { if } x \geq 0 \\
& x F_{ \pm}(x) \geq 0, \quad F_{ \pm}(x) \text { is strictly monotone, if not zero. }
\end{aligned}
$$

According to Eqs. (5) and (6) there are constitutive equations of the rates of partial volumina and mole numbers analogous to Eq. (31).

The main consequence of this section is that partial contact quantities can be associated with corresponding partial parts of the contact surface. The defined contact quantities provide the complete thermodynamic description of non-equilibrium states of discrete systems in an equilibrium environment [3]. However, in the case of interacting non-equilibrium systems, we need some more concepts.

\section{Interacting non-equilibrium systems}

\subsection{Replacement quantities}

We now consider interacting non-equilibrium systems, a situation which differs from the previous one by a non-equilibrium environment instead of an equilibrium one. The first idea one may have is to replace the thermostatic temperature of the equilibrium environment by the contact temperature of the non-equilibrium environment. 
But in this case it can be shown that the sign of the difference of the contact temperatures of the system and its environment does not determine the sign of the heat exchange [12]. That means the heat exchange between these two non-equilibrium systems may not vanish if the contact temperatures of both systems are equal. Therefore, we replace the non-equilibrium environment by that equilibrium one which causes the same net heat exchange as in the original situation. This replacement is possible due to the monotony properties (10) and (11). We call the thermostatic temperature of the replaced equilibrium environment the replacement temperature of the original system's non-equilibrium environment, if the net heat exchange between the system and both environments is identical.

Because the replacement introduces equilibrium environments instead of nonequilibrium ones, the defining inequalities (1-3) are also valid for the replacement quantities: replacement temperature $\vartheta^{*}$, replacement pressure $\pi^{*}$, and replacement chemical potentials $\boldsymbol{v}^{*}$ of the system's non-equilibrium environment,

$$
\begin{aligned}
& \dot{Q}\left(\frac{1}{\Theta}-\frac{1}{\vartheta^{*}}\right) \geq 0, \quad\left(\dot{V}=0, \dot{\mathbf{n}}^{e}=\mathbf{0}\right) \\
& \dot{V}\left(p-\pi^{*}\right) \geq 0, \quad\left(\dot{Q}=0, \dot{\mathbf{n}}^{e}=\mathbf{0}\right) \\
& \dot{\mathbf{n}}^{e} \cdot\left(\boldsymbol{v}^{*}-\boldsymbol{\mu}\right) \geq 0, \quad(\dot{Q}=0, \dot{V}=0) .
\end{aligned}
$$

Here by definition the exchange quantities $\dot{Q}, \dot{V}$, and $\dot{\mathbf{n}}^{e}$ are the same as in the nonequilibrium situation in which two non-equilibrium systems are in contact with each other.

The difference between the inequalities (1) and (34) is as follows: In (1) $T^{*}$ and $\dot{Q}$ are given and $\Theta$ is determined by the zero of $\dot{Q}$, whereas in (34) $\dot{Q}$ and $\Theta$ are given and $\vartheta^{*}$ is determined by these quantities. The same is true for the two other inequalities (2) and (3), (35) and (36). Up to this different interpretation the inequalities (1-3) and (35-36) are formally identical.

Because of the equilibrium concept of replacement quantities, also the constitutive functions $F, G$, and $\mathbf{H}$ in (4-6) are valid for the replacement quantities:

$$
\begin{gathered}
\dot{Q}=F\left(\frac{1}{\Theta}-\frac{1}{\vartheta^{*}}\right), \quad\left(\dot{V}=0, \dot{\mathbf{n}}^{e}=\mathbf{0}\right), \\
\dot{V}=G\left(p-\pi^{*}\right), \quad\left(\dot{Q}=0, \dot{\mathbf{n}}^{e}=\mathbf{0}\right), \\
\dot{\mathbf{n}}^{e}=\mathbf{H}\left(\boldsymbol{v}^{*}-\boldsymbol{\mu}\right), \quad(\dot{Q}=0, \dot{V}=0) .
\end{gathered}
$$

The state space of a discrete system in a non-equilibrium environment should be changed with respect to (12) as

$$
Z=\left\{V, \mathbf{n}, U, \Theta, \xi ; \vartheta^{*}, \pi^{*}, v^{*}\right\}
$$




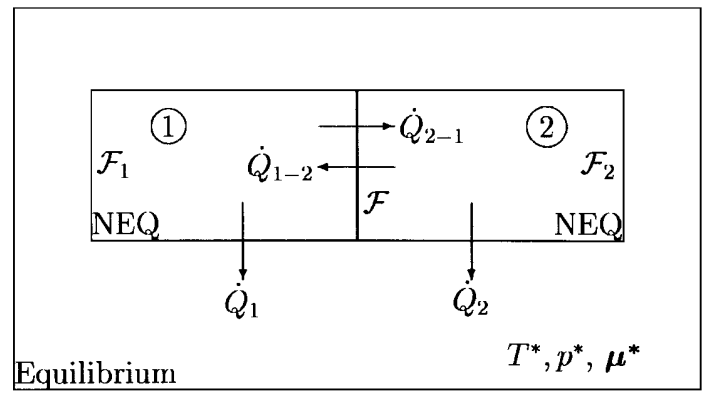

Figure 3 Systems 1 and 2 as parts of a composite system $1 \cup 2$.

Thus in the framework of thermodynamics of discrete systems, we can define all the thermodynamic quantities which are necessary for the complete description of a discrete non-equilibrium system in a non-equilibrium environment.

\subsection{Composite systems}

Two discrete systems 1 and 2 interacting with each other form a composite system $1 \cup 2$, sometimes also called compound system (Fig. 3). It is clear that the phenomenological thermodynamic description of the composite system (by an external observer not knowing that it is composite) contains less information than that of the two subsystems themselves forming the compound system. We denote this fact as compound deficiency. This means that quantities belonging to the composite system differ from those belonging to the sum of both the subsystems forming the composite system. The difference between these quantities is denoted as an excess quantity. In Section 4 the concepts of compound deficiency and excess quantities are worked out in more detail.

3.2.1. The subsystems Suppose that the considered composite system $1 \cup 2$ is composed of two subsystems 1 and 2 which are in interaction with each other and with the environment (marked by ${ }^{*}$ ) which is the same for both (Fig. 3). As usual for Schottky systems, the interaction consists of heat-, power-, and mass-exchange. Here especially the power-exchange is chosen as a volume work for simplification. In general, the subsystems are in non-equilibrium, whereas the environment is presupposed to be in equilibrium because of its reservoir properties.

To describe the exchanges we have to introduce three contact surfaces, one between the subsystems 1 and 2, called $\mathscr{F}$ and two other ones between each of the two subsystems and the environment, denoted by $\mathscr{F}_{1}$ and $\mathscr{F}_{2}$ (see Fig. 3 ). For the three contact surfaces we introduce four partial contact temperatures belonging to the subsystems 1 and $2[13], \Theta_{1}^{\mathscr{F}}, \Theta_{2}^{\mathscr{F}}, \Theta^{\mathscr{F _ { 1 }}}$, and $\Theta^{\mathscr{F _ { 2 }}}$. The meaning of these contact temperatures is clear: $\Theta_{j}^{\mathscr{K}}$ is the partial contact temperature belonging to the contact surface $\mathscr{K}$, and $j$ marks the subsystem, if necessary. The defining inequalities for these contact temperatures are 


$$
\begin{aligned}
& \dot{Q}_{1}\left(\frac{1}{\Theta^{\mathscr{F}_{1}}}-\frac{1}{T^{*}}\right) \geq 0, \\
& \dot{Q}_{2}\left(\frac{1}{\Theta^{\mathscr{F}_{2}}}-\frac{1}{T^{*}}\right) \geq 0,
\end{aligned}
$$

whereas we obtain according to Eq. (34) the following inequalities for the internal contact surface $\mathscr{F}$ :

$$
\begin{aligned}
& \dot{Q}_{1-2}\left(\frac{1}{\Theta_{1}^{\mathscr{F}}}-\frac{1}{\vartheta_{2}}\right) \geq 0, \\
& \dot{Q}_{2-1}\left(\frac{1}{\Theta_{2}^{\mathscr{F}}}-\frac{1}{\vartheta_{1}}\right) \geq 0 .
\end{aligned}
$$

According to Eq. (34), $\vartheta_{1}$ and $\vartheta_{2}$ are the replacement temperatures belonging to the non-equilibrium subsystems 1 and 2 .

Because the contact surface between both the subsystems is an inert one, that means, heat and mass are not absorbed or emitted by this partition ${ }^{2}$, we have $\left(\mathbf{h}_{j}\right.$ are the molar enthalpies of the $\mathrm{j}$-th subsystem)

$$
\dot{Q}_{1-2}+\mathbf{h}_{1} \cdot \dot{\mathbf{n}}_{1-2}^{e}=-\dot{Q}_{2-1}-\mathbf{h}_{2} \cdot \dot{\mathbf{n}}_{2-1}^{e},
$$

and

$$
\dot{\mathbf{n}}_{1-2}^{e}=-\dot{\mathbf{n}}_{2-1}^{e}
$$

Hence, Eq. (44) results in

$$
\left[-\dot{Q}_{1-2}-\left(\mathbf{h}_{1}-\mathbf{h}_{2}\right) \cdot \dot{\mathbf{n}}_{1-2}^{e}\right]\left(\frac{1}{\Theta_{2}^{\mathscr{F}}}-\frac{1}{\vartheta_{1}}\right) \geq 0 .
$$

Because this inequality is valid for arbitrary $\dot{\mathbf{n}}_{1-2}^{e}$, especially also for $\dot{\mathbf{n}}_{1-2}^{e}=\mathbf{0}$, and $\dot{Q}_{1-2}, \Theta_{2}^{\mathscr{F}}$, and $\vartheta_{1}$ are independent of $\dot{\mathbf{n}}_{1-2}^{e}$, we obtain from Eqs. (43) and (47)

$$
\operatorname{sign}\left(\frac{1}{\Theta_{1}^{\mathscr{F}}}-\frac{1}{\vartheta_{2}}\right)=-\operatorname{sign}\left(\frac{1}{\Theta_{2}^{\mathscr{F}}}-\frac{1}{\vartheta_{1}}\right) .
$$

The entropy production generated by the heat exchange between the subsystems of the compound system $1 \cup 2$ is the left-hand side of Eq. (43), or equivalently that of Eq. (44), because the contact surface $\mathscr{F}$ between them is inert. Therefore, the lefthand sides of Eqs. (43) and (44) are equal and Eq. (48) results in

\footnotetext{
${ }^{2}$ For more details on open systems see [14].
} 


$$
\frac{1}{\Theta_{1}^{\mathscr{F}}}-\frac{1}{\vartheta_{2}}=\frac{1}{\vartheta_{1}}-\frac{1}{\Theta_{2}^{\mathscr{F}}} .
$$

By this equation the mean value of the reciprocal replacement temperatures is determined by that of the reciprocal contact temperatures

$$
\frac{1}{\tau^{\mathscr{F}}}:=\frac{1}{2}\left(\frac{1}{\Theta_{1}^{\mathscr{F}}}+\frac{1}{\Theta_{2}^{\mathscr{F}}}\right)=\frac{1}{2}\left(\frac{1}{\vartheta_{1}}+\frac{1}{\vartheta_{2}}\right) .
$$

This relation shows that the replacement temperatures of two non-equilibrium systems being in thermal contact with each other are dependent on each other in contrast to the independent contact temperatures. If one of the contacting subsystems (say 2 ) is in equilibrium with the environment, we obtain from Eq. (50)

$$
\Theta_{2}^{\mathscr{F}}=\vartheta_{2}=T^{*} \rightarrow \vartheta_{1}=\Theta_{1}^{\mathscr{F}}
$$

3.2.2. The composite system In this section we describe the composite system $1 \cup 2$, as if it would not be composed of the two subsytems 1 and 2. This description is of course a more coarse one than that of the two single subsystems in interaction.

As already mentioned, the subsystems of the composite system have two contact surfaces with respect to the environment, $\mathscr{F}_{1}$ and $\mathscr{F}_{2}$, to which the two contact temperatures $\Theta^{\mathscr{F}_{1}}$ and $\Theta^{\mathscr{F _ { 2 }}}$ belong (see Fig. 3). The contact surface of the composite system is $\mathscr{F}_{1} \cup \mathscr{F}_{2}$ to which the contact temperature $\Theta$ belongs. Without restricting generality, we presuppose that

$$
\Theta^{\mathscr{F}_{1}} \leq \Theta^{\mathscr{F}_{2}}
$$

is valid, because this inequality only depends on the numbering of the subsystems. In this case the inequalities (26) and (28) become

$$
\begin{aligned}
& \Theta^{\mathscr{F _ { 1 }}} \leq \Theta \leq \Theta^{\mathscr{F _ { 2 }}} \\
& \sum_{k=1}^{2} \frac{\dot{Q}^{\mathscr{F _ { k }}}}{\Theta^{\mathscr{F _ { k }}}} \geq \frac{\dot{Q}}{\Theta} \geq \frac{\dot{Q}}{T^{*}}, \quad \dot{Q}=\dot{Q}^{\mathscr{\mathscr { F } _ { 1 }}}+\dot{Q}^{\mathscr{\mathscr { F } _ { 2 }}}
\end{aligned}
$$

and we obtain from the inequalities (29) and (30)

$$
\begin{aligned}
& \sum_{k=1}^{2} p^{\mathscr{F}_{k}} \dot{V}^{\mathscr{F}_{k}} \geq p \dot{V} \geq p^{*} \dot{V}, \quad \dot{V}=\dot{V}^{\mathscr{F}_{1}}+\dot{V}^{\mathscr{F}_{2}},{ }^{3} \\
& \sum_{k=1}^{2} \boldsymbol{\mu}^{\mathscr{F}_{k}} \cdot \dot{\boldsymbol{n}}^{e \mathscr{F}_{k}} \leq \boldsymbol{\mu} \cdot \dot{\boldsymbol{n}}^{e} \leq \boldsymbol{\mu}^{*} \cdot \dot{\boldsymbol{n}}^{e}, \quad \dot{\boldsymbol{n}}^{e}=\dot{\boldsymbol{n}}^{e \mathscr{F}_{1}}+\dot{\boldsymbol{n}}^{e \mathscr{F}_{2}} .
\end{aligned}
$$

\footnotetext{
${ }^{3}$ The rates of the partial volumina $\dot{V}^{\mathscr{F} k}$ can be defined properly by using Reynolds transport theorem [4].
} 
Now we have defined both kinds of contact quantities: those for the composite system itself and those for its subsystems. The inequalities (54-56) characterize what we define as compound deficiency. This means that a more detailed description by the subsystems yields different results than a coarse description of the composite system.

\section{Compound deficiency}

\subsection{The inequalities}

We now consider the compound deficiency of the different descriptions of the composite system and its subsystems (Fig. 3).

The power exchange between the two subsystems and the environment is

$$
\dot{W}:=\dot{W}_{1}+\dot{W}_{2}=-\sum_{k=1}^{2} p^{\mathscr{F _ { k }}} \dot{V}^{\mathscr{F _ { k }}}
$$

whereas the power exchange between the composite systems and the environment is, by taking Eq. (55) into account,

$$
\dot{W}_{C S}:=-p \dot{V} \geq \dot{W} .
$$

The energy exchange due to mass exchange between the two subsystems and the environment follows from Eq. (56)

$$
\dot{M}:=\dot{M}_{1}+\dot{M}_{2}=\sum_{k=1}^{2} \boldsymbol{\mu}^{\mathscr{F _ { k }}} \cdot \dot{\boldsymbol{n}}^{e \widetilde{F_{k}}}
$$

The corresponding energy exchange due to mass exchange between the compound system and the environment is

$$
\dot{M}_{C S}:=\boldsymbol{\mu} \cdot \dot{\boldsymbol{n}}^{e} \geq \dot{M}
$$

The last inequality follows from Eq. (56). In section 4.3 we investigate how these compound deficiency inequalities transform to other thermodynamical quantities.

\subsection{Energy and entropy}

The first law of the composite system and its environment is

$$
\dot{U}=\dot{Q}-p \dot{V}+\mathbf{h} \cdot \dot{\mathbf{n}}^{e}, \quad \dot{U}^{*}=\dot{Q}^{*}-p^{*} \dot{V}^{*}+\mathbf{h}^{*} \cdot \dot{\mathbf{n}}^{e *}
$$


The composite system is separated from its environment by an inert partition which is characterized by the following properties [see Eqs. (45), (46)]

$$
\begin{aligned}
& \dot{Q}+\mathbf{h} \cdot \dot{\mathbf{n}}^{e}=-\dot{Q}^{*}-\mathbf{h}^{*} \cdot \dot{\mathbf{n}}^{e *}, \\
& \dot{\mathbf{n}}^{*}=\dot{\mathbf{n}}^{e *}=-\dot{\mathbf{n}}^{e}, \quad \dot{V}=-\dot{V}^{*}, \quad\left(\dot{\mathbf{n}}=\dot{\mathbf{n}}^{e}+\dot{\mathbf{n}}^{i}, \dot{\mathbf{n}}^{i *} \equiv \mathbf{0}\right)
\end{aligned}
$$

(The superscript ${ }^{e}$ denotes external exchange, whereas ${ }^{i}$ marks the change by chemical reactions.)

Therefore $(61)_{2}$ yields

$$
\dot{U}^{*}=-\dot{Q}-p^{*} \dot{V}^{*}-\mathbf{h} \cdot \dot{\mathbf{n}}^{e} .
$$

The reduced heat exchange $\dot{Q} / \Theta$ in Eq. (54) is connected to the entropy rate of a discrete non-equilibrium system [7]

$$
\dot{S}=\frac{1}{\Theta}(\dot{U}+p \dot{V}-\boldsymbol{\mu} \cdot \dot{\mathbf{n}})+\alpha \dot{\Theta}+\boldsymbol{\beta} \cdot \dot{\boldsymbol{\xi}}
$$

Here $\dot{\xi}$ is the time rate of the variables in the state space (12) characterizing irreversible processes. An example for such variables are the chemical reaction speeds. In this case, the $\boldsymbol{\beta}$ are the affinities of the chemical reactions.

The relation (65) represents a non-equilibrium extension of Gibbs fundamental equation which for the equilibrium environment is as follows:

$$
\dot{S}^{*}=\frac{1}{T^{*}}\left(\dot{U}^{*}+p^{*} \dot{V}^{*}-\boldsymbol{\mu}^{*} \cdot \dot{\mathbf{n}}^{*}\right)
$$

The rates $\dot{\Theta}$ and $\dot{\boldsymbol{\xi}}$ describe non-equilibrium. The non-equilibrium entropy is a function $S(U, V, \mathbf{n}, \Theta, \xi)$ on the state space [7]. The equilibrium entropy $S^{*}\left(U^{*}, V^{*}, \mathbf{n}^{*}\right)$ depends on the equilibrium variables of the environment.

Introducing Eqs. (61) 1 and (64) into Eqs. (65) and (66) we obtain

$$
\begin{aligned}
& \dot{S}=\frac{1}{\Theta}\left(\dot{Q}+\mathbf{h} \cdot \dot{\mathbf{n}}^{e}-\boldsymbol{\mu} \cdot \dot{\mathbf{n}}\right)+\alpha \dot{\Theta}+\boldsymbol{\beta} \cdot \dot{\boldsymbol{\xi}}, \\
& \dot{S}^{*}=\frac{1}{T^{*}}\left(-\dot{Q}-\mathbf{h} \cdot \dot{\mathbf{n}}^{e}+\boldsymbol{\mu}^{*} \cdot \dot{\mathbf{n}}^{e}\right) .
\end{aligned}
$$

By the molar entropies

$$
\Theta \mathbf{s}:=\mathbf{h}-\boldsymbol{\mu}, \quad T^{*} \mathbf{s}^{*}:=\mathbf{h}^{*}-\boldsymbol{\mu}^{*},
$$

Eqs. (67) and (68) result in 


$$
\begin{aligned}
& \dot{S}=\frac{1}{\Theta}\left(\dot{Q}+\Theta \mathbf{s} \cdot \dot{\mathbf{n}}^{e}-\boldsymbol{\mu} \cdot \dot{\mathbf{n}}^{i}\right)-\alpha \dot{\Theta}+\boldsymbol{\beta} \cdot \dot{\boldsymbol{\xi}}, \\
& \dot{S}^{*}=\frac{1}{T^{*}}\left(-\dot{Q}-\Theta \mathbf{s} \cdot \dot{\mathbf{n}}^{e}+\left(\boldsymbol{\mu}^{*}-\boldsymbol{\mu}\right) \cdot \dot{\mathbf{n}}^{e}\right) .
\end{aligned}
$$

Presupposing additivity of entropies we obtain for the entropy rate of the isolated total system

$$
\begin{aligned}
\dot{S}^{t o t}=\dot{S}+\dot{S}^{*}= & \left(\frac{1}{\Theta}-\frac{1}{T^{*}}\right)\left(\dot{Q}+\Theta \mathbf{s} \cdot \dot{\mathbf{n}}^{e}\right) \\
& +\frac{1}{T^{*}}\left(\boldsymbol{\mu}^{*}-\boldsymbol{\mu}\right) \cdot \dot{\mathbf{n}}^{e}-\frac{\boldsymbol{\mu}}{\Theta} \cdot \dot{\mathbf{n}}^{i}+\alpha \dot{\Theta}+\boldsymbol{\beta} \cdot \dot{\boldsymbol{\xi}} \geq 0
\end{aligned}
$$

Here the inequality is caused by the second law valid for isolated systems for which entropy rate and entropy production are identical.

If there is no heat and mass exchange between the system and its environment, (72) results in the entropy production of the system under consideration

$$
-\frac{\boldsymbol{\mu}}{\Theta} \cdot \dot{\mathbf{n}}^{i}+\alpha \dot{\Theta}+\boldsymbol{\beta} \cdot \dot{\boldsymbol{\xi}} \geq 0
$$

Because this entropy production is independent of the intensive variables of the equilibrium environment, from Eq. (72) the following inequality results:

$$
\left(\frac{1}{\Theta}-\frac{1}{T^{*}}\right)\left(\dot{Q}+\Theta \mathbf{s} \cdot \dot{\mathbf{n}}^{e}\right)+\frac{1}{T^{*}}\left(\boldsymbol{\mu}^{*}-\boldsymbol{\mu}\right) \cdot \dot{\mathbf{n}}^{e} \geq 0 .
$$

If $\dot{\mathbf{n}}^{e}=\mathbf{0}$, we obtain Eq. (1), if $T^{*}=\Theta$, Eq. (3) follows. Thus, the defining inequalities are rediscovered.

For discussing compound deficiency in more detail we consider a special example in the next section.

\subsection{Example: An endoreversible system}

In the sequel we consider an endoreversible system which by definition consists of subsystems being in different equilibria [15]. Because these equilibria are different, irreversible processes take place between the subsystems. The use of endoreversible systems represents an analogue for discrete systems of the often accepted hypothesis of local equilibrium of classical irreversible thermodynamics [16-22]. Using it, points of the non-equilibrium state space are associated with points of equilibrium subspace by means of a projection. Thus, we suppose that a non-equilibrium state of each discrete system is associated with an equilibrium state of the accompanying reversible process. 
In an endoreversible system all contact quantities of the subsystems are identified with equilibrium bulk values

$$
\Theta^{\mathscr{F}_{j}}=\Theta_{j}^{\mathscr{F}}=\vartheta_{j}=: T_{j}, \quad j=1,2 .
$$

For local equilibrium Eq. (50) is satisfied tritely.

In case of an endoreversible system, the state space is much smaller than that in nonequilibrium (12) [3]:

$$
Z^{e q}=\{V, \mathbf{n}, T\}
$$

For the two subsystems in consideration, the local accompanying states are

$$
Z_{j}^{e q}=\left\{V_{j}, \mathbf{n}_{j}, T_{j}\right\}, \quad T_{1} \neq T_{2}, \quad V_{1} \neq V_{2}, \quad \mathbf{n}_{1} \neq \mathbf{n}_{2} .
$$

According to Eq. (77), the entropies of the equilibrium subsystems 1 and 2 are (Fig. 3):

$$
\begin{aligned}
& \dot{S}_{1}=\frac{1}{T_{1}} \dot{Q}_{1}+\mathbf{s}_{1} \cdot \dot{\mathbf{n}}_{1}^{e}+\frac{\dot{Q}_{1-2}}{T_{1}}+\mathbf{s}_{1} \cdot \dot{\mathbf{n}}_{1-2}^{e} \\
& \dot{S}_{2}=\frac{1}{T_{2}} \dot{Q}_{2}+\mathbf{s}_{2} \cdot \dot{\mathbf{n}}_{2}^{e}+\frac{\dot{Q}_{2-1}}{T_{2}}+\mathbf{s}_{2} \cdot \dot{\mathbf{n}}_{2-1}^{e} .
\end{aligned}
$$

Because the partition between the two subsystems is an inert one, we obtain, according to Eqs. (45) and (46), for the heat exchanges of both the subsystems:

$$
\dot{Q}_{1-2}+\mathbf{h}_{1} \cdot \dot{\mathbf{n}}_{1-2}^{e}=-\dot{Q}_{2-1}-\mathbf{h}_{2} \cdot \dot{\mathbf{n}}_{2-1}^{e} \quad \dot{\mathbf{n}}_{2-1}^{e}=-\dot{\mathbf{n}}_{1-2}^{e}
$$

Inserting Eq. (80) into Eq. (79) yields

$$
\begin{aligned}
\dot{S}:= & \dot{S}_{1}+\dot{S}_{2}=\frac{\dot{Q}_{1}}{T_{1}}+\frac{\dot{Q}_{2}}{T_{2}}+\mathbf{s}_{1} \cdot \dot{\mathbf{n}}_{1}^{e}+\mathbf{s}_{2} \cdot \dot{\mathbf{n}}_{2}^{e} \\
& +\left(\frac{1}{T_{1}}-\frac{1}{T_{2}}\right) \dot{Q}_{1-2}+\left(\mathbf{s}_{1}-\mathbf{s}_{2}\right) \cdot \dot{\mathbf{n}}_{1-2}^{e}+\frac{1}{T_{2}}\left(\mathbf{h}_{2}-\mathbf{h}_{1}\right) \cdot \dot{\mathbf{n}}_{1-2}^{e} .
\end{aligned}
$$

The composite system $1 \cup 2$ is an endoreversible non-equilibrium system whose entropy rate is, according to Eq. (70),

$$
\dot{S}_{C S}:=\frac{1}{\Theta} \dot{Q}+\mathbf{s} \cdot \dot{\mathbf{n}}^{e}
$$

For the internal energy we obtain, according to Eq. (61),

$$
\dot{U}_{1}=\dot{Q}_{1}-p_{1} \dot{V}_{1}+\mathbf{h}_{1} \cdot \dot{\mathbf{n}}_{1}^{e}+\dot{Q}_{1-2}-p_{1} \dot{V}_{1-2}+\mathbf{h}_{1} \cdot \dot{\mathbf{n}}_{1-2}^{e},
$$




$$
\dot{U}_{2}=\dot{Q}_{2}-p_{2} \dot{V}_{2}+\mathbf{h}_{2} \cdot \dot{\mathbf{n}}_{2}^{e}+\dot{Q}_{2-1}-p_{2} \dot{V}_{2-1}+\mathbf{h}_{2} \cdot \dot{\mathbf{n}}_{2-1}^{e}
$$

Presupposing the additivity of partial energies yields, if Eq. (80) is taken into account,

$$
\begin{aligned}
\dot{U} & :=\dot{U}_{1}+\dot{U}_{2} \\
& =\dot{Q}_{1}+\dot{Q}_{2}-p_{1} \dot{V}_{1}-p_{2} \dot{V}_{2}+\mathbf{h}_{1} \cdot \dot{\mathbf{n}}_{1}^{e}+\mathbf{h}_{2} \cdot \dot{\mathbf{n}}_{2}^{e}-\left(p_{1}-p_{2}\right) \dot{V}_{1-2}
\end{aligned}
$$

For the endoreversible non-equilibrium composite system we obtain from Eq. (61):

$$
\dot{U}_{C S}=\dot{Q}-p \dot{V}+\mathbf{h} \cdot \dot{\mathbf{n}}^{e}
$$

A comparison of entropies (82) and energies (86) of the composite system with those of the subsystems (81) and (85) shows that they are different:

$$
\dot{S}_{C S} \neq \dot{S}, \quad \dot{U}_{C S} \neq \dot{U}
$$

This fact is what we denoted by compound deficiency. Also the inequalities (58) and (60) are caused by compound deficiency. The differences between the quantities belonging to the composite system and those belonging to the subsystems are discussed in the next section.

\subsection{Excess quantities}

4.4.1. Excess power exchange, excess mass exchange To describe compound deficiency in more detail we introduce excess quantities. If we denote a special quantity by $\square$, the corresponding excess quantity $\square^{E X}$ is defined by

$$
\sum_{k} \square_{k}+\square^{E X}:=\square_{C S} .
$$

We now discuss some of these excess quantities.

From Eq. (55) we obtain the excess power exchange

$$
\dot{W}^{E X}=-p \dot{V}+p_{1} \dot{V}_{1}+p_{2} \dot{V}_{2} \geq 0
$$

If the discrete system in consideration is non-composed, that means, if $p_{1}=p_{2}=p$ is valid, we obtain $\dot{W}^{E X}=0$.

The excess energy exchange due to mass exchange is, according to Eq. (56),

$$
\dot{M}^{E X}=\boldsymbol{\mu} \cdot \dot{\mathbf{n}}^{e}-\boldsymbol{\mu}_{1} \cdot \dot{\mathbf{n}}_{1}^{e}+\boldsymbol{\mu}_{2} \cdot \dot{\mathbf{n}}_{2}^{e} \geq 0
$$

If the discrete system in consideration is non-composed, that means, if $\boldsymbol{\mu}_{1}=\boldsymbol{\mu}_{2}=\boldsymbol{\mu}$ is valid, we obtain $\dot{M}^{E X}=0$. 
4.4.2. Excess energy According to Eq. (88) the rate of the excess energy is, using Eqs. (86) and (85),

$$
\begin{aligned}
\dot{U}^{E X}:= & \dot{U}_{C S}-\dot{U}_{1}-\dot{U}_{2}=\dot{Q}-p \dot{V}+\mathbf{h} \cdot \dot{\mathbf{n}}^{e} \\
& -\dot{Q}_{1}-\dot{Q}_{2}+p_{1} \dot{V}_{1}+p_{2} \dot{V}_{2}-\mathbf{h}_{1} \cdot \dot{\mathbf{n}}_{1}^{e}-\mathbf{h}_{2} \cdot \dot{\mathbf{n}}_{2}^{e}+\left(p_{1}-p_{2}\right) \dot{V}_{1-2} .
\end{aligned}
$$

Taking Eqs. (17) 3 and (89) into account we obtain:

$$
\dot{U}^{E X}=\dot{W}^{E X}+\left(\mathbf{h}-\mathbf{h}_{1}\right) \cdot \dot{\mathbf{n}}_{1}^{e}+\left(\mathbf{h}-\mathbf{h}_{2}\right) \cdot \dot{\mathbf{n}}_{2}^{e}+\left(p_{1}-p_{2}\right) \dot{V}_{1-2} .
$$

If the discrete system under consideration is non-composed, that means, if $\mathbf{h}_{1}=$ $\mathbf{h}_{2}=\mathbf{h}$ and $p_{1}=p_{2}$ are valid, we obtain $\dot{U}^{E X}=0$.

4.4.3. Excess entropy According to Eq. (88), the rate of the excess entropy is, using Eqs. (82) and (81),

$$
\begin{aligned}
\dot{S}^{E X}:= & \dot{S}_{C S}-\dot{S}_{1}-\dot{S}_{2}=\frac{1}{\Theta} \dot{Q}+\mathbf{s} \cdot \dot{\mathbf{n}}^{e}-\frac{\dot{Q}_{1}}{T_{1}}-\frac{\dot{Q}_{2}}{T_{2}} \\
& -\mathbf{s}_{1} \cdot \dot{\mathbf{n}}_{1}^{e}-\mathbf{s}_{2} \cdot \dot{\mathbf{n}}_{2}^{e}-\left(\frac{1}{T_{1}}-\frac{1}{T_{2}}\right) \dot{Q}_{1-2} \\
& -\left(\mathbf{s}_{1}-\mathbf{s}_{2}\right) \cdot \dot{\mathbf{n}}_{1-2}^{e}-\frac{1}{T_{2}}\left(\mathbf{h}_{2}-\mathbf{h}_{1}\right) \cdot \dot{\mathbf{n}}_{1-2}^{e} .
\end{aligned}
$$

Using Eqs. (69) we obtain after a short calculation:

$$
\begin{aligned}
\dot{S}^{E X}= & \left(\frac{1}{\Theta}-\frac{1}{T_{1}}\right) \dot{Q}_{1}+\left(\frac{1}{\Theta}-\frac{1}{T_{2}}\right) \dot{Q}_{2} \\
& +\left(\mathbf{s}-\mathbf{s}_{1}\right) \cdot \dot{\mathbf{n}}_{1}^{e}+\left(\mathbf{s}-\mathbf{s}_{2}\right) \cdot \dot{\mathbf{n}}_{2}^{e} \\
& -\left(\frac{1}{T_{1}}-\frac{1}{T_{2}}\right)\left(\dot{Q}_{1-2}+\mathbf{h}_{1} \cdot \dot{\mathbf{n}}_{1-2}^{e}\right)-\left(\frac{\boldsymbol{\mu}_{2}}{T_{2}}-\frac{\boldsymbol{\mu}_{1}}{T_{1}}\right) \cdot \dot{\mathbf{n}}_{1-2}^{e} .
\end{aligned}
$$

If the discrete system in consideration is non-composed, that means, if $T_{1}=T_{2}=\Theta$, $\mathbf{s}_{1}=\mathbf{s}_{2}=\mathbf{s}$ and $\boldsymbol{\mu}_{1}=\boldsymbol{\mu}_{2}$ are valid, we obtain $\dot{S}^{E X}=0$.

\section{Summary}

A discrete system may be composed of subsystems interacting with each other or may be non-composed. Therefore, the description of the discrete system under consideration depends on the information one has about the system. Clear is that both the descriptions are different, because different levels of information result in different contact quantities between the considered discrete system and its equilibrium environment. So, e.g., the contact temperatures depend on the description: if the 
discrete system is considered as being non-composed, the contact temperature is that of the whole contact surface between the discrete system and its environment. If the discrete systems is described as a composite system, the contact temperatures of the partial contact surfaces between the subsystems of the composite system and the environment play a role.

The difference of these descriptions is a general feature in thermodynamics of discrete systems which we characterize by the concept of compound deficiency. Starting out with the additivity of partial exchange quantities, the compound deficiency results in different net exchange quantities and different energies and entropies depending on the description of the considered discrete system as being composed or not. The thermodynamic consistency between the different descriptions is achieved by introducing excess quantities.

As it is shortly discussed, the concept of compound deficiency can be extended to the contact of two discrete non-equilibrium systems by introducing replacement quantities instead of contact quantities.

The excess quantities of energy, entropy, and power and the energy exchanges are calculated and discussed for an endoreversible compound system.

\section{Acknowledgements}

Support of the Estonian Science Foundation under contracts No. 2247, 3203, of the DAAD grant A/97/17268 (A.B.), and financial support by VISHAY Comp. (D91085 Selb, Germany) (W.M.) is gratefully acknowledged.

\section{References}

[1] Wilmanski, K., Thermomechanics of Continua, chap. 4, Springer, Berlin, 1988.

[2] Jou, D., Casas-Vazquez, J., Lebon, G., Extended Irreversible Thermodynamics, 3rd ed., chap. 2, Springer, Berlin, 2001.

[3] Muschik, W., Papenfuss, C., Ehrentraut, H., Concepts of Continuum Thermodynamics, chap. 3, Technische Universität Berlin, Kielce University of Technology, Kielce, Poland, 1997.

[4] Muschik, W., Papenfuss, C., Ehrentraut, H., A sketch of continuum thermodynamics, J. Non-Newtonian Fluid Mech., 96 (2001), 255.

[5] Muschik, W., Aspects of Non-Equilibrium Thermodynamics, sec. 1.1, World Scientific, Singapore, 1990.

[6] Truesdell, C., Bharatha, S., The Concepts and Logic of Classical Thermodynamics as a Theory of Heat Engines, p. xiii, Springer, New York, 1977.

[7] Muschik, W., Fundamentals of non-equilibrium thermodynamics, in: Non-Equilibrium Thermodynamics with Application to Solids, 1-63, Ed. Muschik, W., Springer, Wien, 1993.

[8] Schottky, W., Thermodynamik, sec. 1, paragr. 1, Springer, Berlin, 1929, reprint 1973.

[9] Muschik, W., Empirical foundation and axiomatic treatment of non-equilibrium temperature, Arch. Rat. Mech. Anal., 66 (1977), 379.

[10] Muschik, W., Brunk, G., A concept of non-equilibrium temperature, Int. J. Engng. Sci., 15 (1977), 377. 
[11] Muschik, W., Fundamental remarks on evaluating dissipation inequalities, in: Recent Developments in Nonequilibrium Thermodynamics, 388-397, Eds. Casas-Vazquez, J., Jou, D., Lebon, G., Lecture Notes in Physics 199, Springer, Berlin, 1984.

[12] Muschik, W., Entropies of heat conducting discrete or multi-temperature systems with use of non-equilibrium temperatures, Int. J. Engng. Sci., 18 (1980), 1399.

[13] Muschik, W., Domínguez-Cascante, R., On extended thermodynamics of discrete systems, Physica A, 233 (1996), 523.

[14] Muschik, W., Gümbel, S., Does Clausius' inequality exist for open systems?, J. NonEquilib. Thermodyn., 24 (1999), 97-106.

[15] Hoffmann, K.-H., Burzler, J.M., Schubert, S., Endoreversible thermodynamics, J. NonEquilib. Thermodyn., 22 (1997), 311.

[16] Keller, J.U., Ein Beitrag zur Thermodynamik fluider Systeme, Physica, 53 (1971), 602.

[17] Muschik, W., Fundamentals of dissipation inequalities, J. Non-Equilib. Thermodyn., 4 (1979), 277.

[18] Kestin, J., Local-equilibrium formalism applied to mechanics of solids, Int. J. Solids Struct., 29 (1992), 1827.

[19] Kestin, J., Internal variables in the local-equilibrium approximation, J. Non-Equilib. Thermodyn., 18 (1993), 360.

[20] Maugin, G.A., Muschik, W., Thermodynamics with internal variables. Part 1. General concepts, J. Non-Equilib. Thermodyn., 19 (1994), 217.

[21] Maugin, G.A., The Thermomechanics of Nonlinear Irreversible Behavior. An Introduction, chap. 9, World Scientific, Singapore, 1999.

[22] De Groot, S.R., Mazur, P., Non-Equilibrium Thermodynamics, chap. III, NorthHolland, Amsterdam, 1961.

Paper received: 2003-08-18

Paper accepted: 2004-04-25 
Brought to you by | Technische Universität Berlin 